\title{
A Social Network Environment Based Analysis for Construction of Cancer Care
}

\author{
Ilindra. Lakshmi Chandralekha ${ }^{1}$, Dr. P. Siva Kumar ${ }^{2}$ \\ ${ }^{1,2}$ Computer Science and Engineering, Rise Group of Institutions, Ongole, India
}

\begin{abstract}
Social media provides rich sources of personal information and community interaction which can be linked to aspect of mental health. In this paper we investigate manifest properties of textual messages, including latent topics, psycholinguistic features, and authors' health, of a large corpus of blog posts, to analyze the aspect of social capital in social media communities. Using data collected from Live Journal, we find that bloggers with lower social capital have fewer positive moods and more negative moods than those with higher social capital. It is also found that people with low social capital have more random mood swings over time than the people with high social capital. Significant differences are found between low and high social capital groups when characterized by a set of latent topics and psycholinguistic features derived from blogposts, suggesting discriminative features, proved to be useful for classification tasks. Good prediction is achieved when classifying among social capital groups using topic and linguistic features, with linguistic features are found to have greater predictive power than latent topics. The significance of our work lies in the importance of online social capital to potential construction of automatic healthcare monitoring systems. We further establish the link between health and social capital in online communities, suggesting the foundation of new systems to monitor online mental well-being.
\end{abstract}

Keywords: Social Network, Structure, ties, egocentric network, cancer, social support, Mental QOL

\section{Introduction}

Since the 1970s, there has been a dramatic increase of interest in the concept of social support and its impact on mental health and well-being. As health management is largely a social process, social networks have been theorized to impact health positively through five mechanisms: social support, social influence, access to resources, social participation, and person-to-person interaction. Berkman [4] states three categories of social networks that influences health - - (a) physiological stress responses; (b) psychological states including self-esteem and self-efficacy; and (c) health behaviors, both positive (e.g., exercise, health service utilization, medical adherence) and negative (e.g., tobacco consumption, overeating)". [46]

Socially-isolated individuals are less able to buffer the impact of health stressors than others and consequently are at greater risk of negative health effects such as quality of life (QOL) illness or death. While this have been replicated multiple times in several populations $[14,30]$, very few research studies focus on the significance of social structure, social position and social ties plus their impact on QOL. A key principle in this study is that understanding the relationship between social network properties and Global Mental QOL of people with cancer which is instrumental in the management of QOL by health professionals, practitioners and consumers. The Global Mental Health index indicates (i) the extent to which cancer patients are satisfied with their social activities and relationships; (ii) how often have they been bothered by emotional problems such as feeling anxious, depressed or irritable; and (iii) how well they carried out their usual social activities and roles including activities at home, at work or in communities, including carrying out their responsibilities as a parent, spouse, employee, friend, etc. Accordingly, the motivating questions are: what forms of network structure would impact Global Mental QOL positively? What network position influences Global Mental
QOL? Does diversity of peers have any impact on Global Mental QOL? How does strength of ties impact the Global Mental QOL?

We develop a theoretical framework based on the theory of structural holes, strength of weak tie theory and Freeman's concept of centrality for understanding how social support impacts Global Mental QOL in cancer patient's life. In the following sections we review literature on effects of social support in the health context along with the research model and hypotheses. We then describe the domain for our research followed by results, discussion and conclusion.

\section{Theoretical Foundations}

The 1960s and 70s witnessed a shift from focusing on material gain to achieving social and psychological needs as government in western society directed policy towards re establishing welfare, after World War II. Some major surveys were undertaken in the United States to investigate correlation between objective and subjective conditions and level of wellbeing in population and resulted in developing QOL Scale by John Flanagan [7] in the mid 1970's. QOL scale items including 15 components, which were classified into five categories, namely (i) marital and physical wellbeing (ii)relationship with other people, (iii) social, community and civic activities (iv) personal development and fulfilment and (v)recreation.

It is difficult to define meaning of 'Quality of Life' and quantify it by comparing one individual with another while measurement may not be important from the other patient's point of view, plus QOL might vary significantly with time. Therefore, QOL can only be defined and measured in individual terms, and depends on present lifestyle, past experience, hopes for the future, dreams and ambitions as well as impact of sickness and treatment. A 'good' QOL is usually expressed in terms of satisfaction, contentment, 


\section{International Journal of Science and Research (IJSR) \\ ISSN (Online): 2319-7064 \\ Index Copernicus Value (2013): 6.14 | Impact Factor (2014): 5.611}

happiness and fulfilment and the ability to cope. Impact of an illness and the way people cope with a sickness, depends on their current situation and ability to cope. Individuals would be able to cope with a disease easier when they feel well, content or satisfied in life and vice versa. For improving QOL, expectations, goals and ambitions might need to be reduced by making them more realistic and appropriate. Some individuals have a good QOL although they suffer major problems such as serious physical illness or poor social conditions. They achieve that by reducing their expectations and are satisfied with what they have, or by being able to overcome problems with personal growth and development. Although frequently perceived by the individuals themselves, to increase QOL of an individual, energy is required which can either be self-generated or comes from outside and others in the shape of support from interaction network members [11].

Support network satisfaction is linked with positive health outcomes so it may help to decrease uncertainty, which often comes together with stressful events and may assist in better coping with problems [55]. For instance it was found that breast cancer patients who had an internal locus of control took more responsibility for their own health care regimes due to being more satisfied with their support networks [50]. The majority of studies found that discerned support is more strongly associated with well-being and mental health than actual support $[24,36]$.

Psychosocial involvements are known as an important and cost effective factor in treatment of many chronic diseases [21]. Social support has an extraordinary significance in dealing with psychological difficulties such as anxiety and depression, which equally are common in cancer patients. For instance, in a study of patients with diverse types of cancer, the frequency of psychological illness one year after diagnosis was $31.8 \%$ while patients with low social support grades were reported to have depression [45]. Depression was reported as a significant predictor for QOL in breast cancer patients [56]. Consequently, social support plays a crucial role in dealing with detriments that result from cancer and its treatment [17].

Four different functions of social support have been described: emotional support, instrumental support, appraisal support, and informational support, where family members offer emotional support like esteem, trust, concern, and listening. Instrumental support consists of aid in kind, money, labor, and time. Peers offer appraisal support that enhances the individual's self-esteem. Finally, informational support includes advice, suggestions for problem solving, directives, and information [22, 32]. Structural characteristics of social support refer to the structure of a social network or sources of support while functional characteristics refer to the provision of particular resources or types of support $[47,34]$.

In the domain of QOL related to cancer patients, those who are socially isolated are more likely to have a higher risk of mortality after a diagnosis of cancer due to lack of access to care, specifically beneficial care giving from friends, relatives that are embedded in one's social networks. Cancer patients require social support including instrumental, emotional and informational support as well as social networks.
Instrumental support would assist cancer patients to get to medical appointments, remind them to take medications and assist with nutrition and mobility. This would protect patients against disability plus help to go through the treatment process, while they are mentally and physically under pain and pressure and might unable to perform these tasks personally. Social emotional support, often provided by a confidant, may reduce stress, which might improve immunosurveillance against cancer re-appearance. Investigators have suggested that social emotional support may be more critical than instrumental support for breast cancer survival [33].

In consideration of above discussion, the conceptual relationship model between social network, support and wellbeing is demonstrated in the following figure.

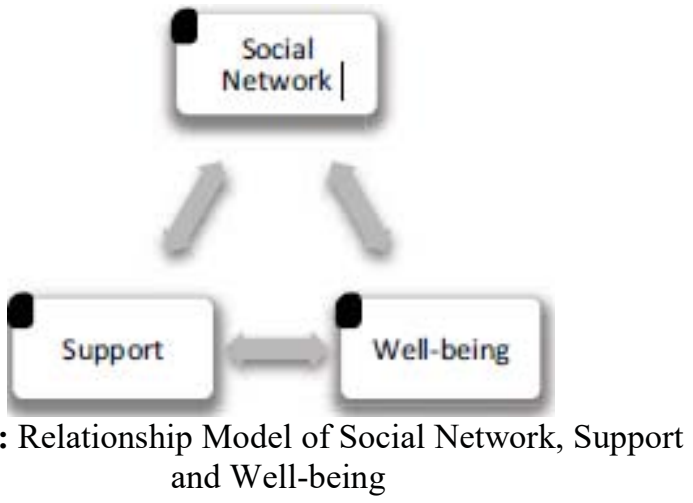

Towards a Social Network based Model for QOL

The study on effects of social networks on health began in the 1970s through the work of researchers such as Cassel, Cobb, and Berkman, who proven empirically that social networks could impact mortality [12, 13, 3, 5, 29]. In the light of above discussion, social network theories such as Freeman's degree centrality [18], Granovetter's strength of strong ties [23] are particularly relevant and have been utilized in developing a theoretical research model for this study.

In Freeman's concept of actor level centrality, Degree Centrality is defined as the number of connections or ties one has in their personal network [18]. Social support represented by the number of ties which an individual has, is clearly psychologically helpful for cancer patients, and for those with other diseases, to benefit their health and wellbeing, including reduction of stress that arises from illness, treatment and adaptability and adjustment to the disease [2, $14,28,54]$.

In the first major study on social relationships and mortality, Berkman \& Syme [3] reported data from a probability sample of 2229 men and 2496 women, who were aged 30-69 when interviewed in 1965 in Alameda County, California. The data included measures of the presence or absence of four types of social ties in their marriage, contacts with extended family and friends, church membership, and other formal and informal group affiliations. Each of these four types of social relationships predicted the rate of mortality over the succeeding nine years. Each effect was independent of the others while intimate ties of marriage and contact with family 


\section{International Journal of Science and Research (IJSR) \\ ISSN (Online): 2319-7064 \\ Index Copernicus Value (2013): 6.14 | Impact Factor (2014): 5.611}

and friends were, the stronger the predictors of mortality rates than the church and group membership predictors. Similar outcomes reported by other studies $[29,5]$. On this basis, we hypothesize the importance of the number of ties in the following:

H1: At the actor level, an individual's network size or Degree centrality is associated with Global Mental QOL

Our second hypothesis is based on the theory of the strength of ties. The strength of a tie is a combination of the amount of time, the emotional intensity, the intimacy (mutual confiding), and the reciprocal services which characterize the tie" [23]. Strong ties provide emotional aid, small services, and friendship thus impact Mental QOL dimensions as explained earlier. Community ties with friends, family members and relatives provide social support that exceeds the need for reciprocity and creates social capital which eases QOL, including handling of significant life events, decreasing uncertainties and leveraging opportunities [31]. Furthermore, their impact on household's survival, expansion, and reproduction have been demonstrated in varies research studies [39].

Numerous studies indicate that people with spouses, friends, and family members who provide psychological and material resources are in better health than those with fewer supportive social contacts $[6,35,37]$. Networks of small size, strong ties, high density, high homogeneity, and low dispersion appear to be helpful in maintaining social identity and hence health and well-being outcomes when these are promoted by maintenance of the networks. However, change in social roles and identities, and hence health and well-being during such change, is facilitated by larger networks with weaker ties, lower density, and greater social and cultural heterogeneity $[26,27,40,48,49,51]$. We therefore hypothesize that tie strength is crucial for Mental QOL and thus state the following:

H2: At the dyadic level, strength of tie within an individual's network is associated with Global Mental QOL

According to Burt's structural holes theory, an individual who bridges two or more components (e.g. cliques) within the network is said to occupy a structural hole ${ }^{\text {' and is thus }}$ very efficiently situated. Analogous to brokerage-based roles, this position is extremely conducive in terms of accessing novel, diverse sources of information and social groups [8]. Depending on context, one may also control the flow of information or other resources in such a situation. Here, the efficiency ' index is correlated to number of non-redundant contacts. So when the number of non-redundant contacts increases, efficiency increases as the actor will have more opportunities to connect with new people. Hence new information benefits would consequently increase in level of awareness and knowledge health related information may come to fruition. Increase in quality of information would reduce uncertainty and stress which is caused by uncertainty. Reducing uncertainty in a cancer patient would influence emotional well-being. Enhanced emotional well-being is seen as a main contributor to increase self-esteem, fulfillment, coping ability, performance and longevity [18]. Therefore, increase in emotional well-being would have an association with Global Mental QOL of cancer patients. We can now postulate the following hypothesis:

H3: At the actor's positional level, efficiency of an individual's network is associated with Global Mental QOL

- Cetralization of a network is a degree of how central its most central node is in relation to how central all the other nodes are" [18]. Centralization doesn't focus on the relative status of points but to the overall interconnection or integration of the network [43]. Previous studies have shown that network centrality would impact decision-making [20] and that denser networks are favorable for knowledge sharing [15]. This would impact emotional well-being of a cancer patient and thus an individual's Global Mental QOL. Therefore the following hypothesis is stated:

\section{H4: At the network level, density of an individual's network is associated with Global Mental QOL}

As stated previously, according to Burt [8] an individual derives information and resource benefits due to the network position the individual is located in. This brings about diversity because of the structural hole that the individual occupies. Interestingly, it also means that at the tie-level, there is diversity in the information or resource that is channelled through the tie. Such diversity includes functional diversity, the extent to which an individual has diverse ties with individuals from varying occupations or functions. Functional diversity may thus potentially influence Global Mental QOL of the individual. Most studies illustrate the significance of social support provided by a partner, family members and other relatives and its positive supportive impact on QOL.

On the contrary, Wortman and Dunkel-Schetter [53] indicated that cancer patients can be victimized by their family members and friends. They mentioned that cancer creates two conflicting reactions including fear and disgust thus it may create ambivalence toward the patient and anxiety about interacting with that individual. Subsequently, some people may physically avoid any interaction with the cancer patient or may openly evade communication about the illness. These inconsistencies in conduct (positive verbal and negative nonverbal behaviour) can lead the patient to feel rejected and abandoned by family members and friends. Wine field and Katsikitis [52] found that for survivors of myocardial infarction, doctors and other health professionals were the main sources of informational and problemorientated support. The result from Dakof and Taylor [16] research study is aligned with previous studies and indicates that patients intend their professional contacts to be qualified, informative and empathetic [42]. Additionally, diversity of ties (in terms of network range or ties to diverse knowledge groups) would positively be associated with easiness of knowledge transfer. We therefore hypothesize.

\section{Volume 4 Issue 11, November 2015}




\section{International Journal of Science and Research (IJSR) ISSN (Online): 2319-7064 \\ Index Copernicus Value (2013): 6.14 | Impact Factor (2014): 5.611}

H5: At the dyadic level, functional diversity of an individual's network is associated with Global Mental QOL

Constraint dictates the extent to which an individual's opportunities are limited by investing the bulk of his or her network time and energy in relationships that lead back to the single contact $[8$, p. 55]. Constraint basically measures the degree to which an individual's contacts are connected to each other and is therefore describes redundancy of contacts. According to Hanneman [25], constraint also measures the extent to which an individual is connected to others who are connected to one another. Therefore if one has many connections to others who in turn has many connections to more others, then the individual is quite constrained. At organizational levels, individuals with high constraint indices are unable to formulate novel ideas because of the redundant nature of information which is obtained from a densely connected group of individuals. Prior research studies consistently show that high efficiency and low constraint indices are useful indicators of an individual's ability to develop good ideas [9], get ahead" in terms of job performance and promotion $[8,10]$ and enjoy greater career mobility [41]. In this research context, high constraint, denser network, indicates frequent, close, collaborative and potentially confiding relationships between cancer patients and their contacts, and between contacts themselves. We therefore hypothesize the following:

H6: At the actor's positional level, constraint of an individual's network is associated with Global Mental QOL

Resulting from H1-H6, the theoretical research model for studying Social Network's impact on QOL of cancer patients is demonstrated in the following figure.

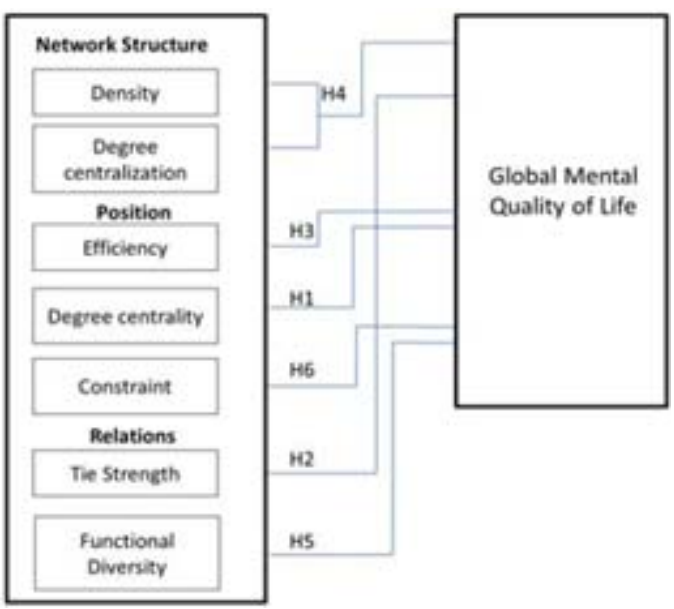

Figure 2: Research Model for Studying Social Network's Impact on Global Mental QOL of Cancer Patients

\section{Methodology}

As the subject of this study is evaluating Global Mental QOL cancer patients and examine impact of social support or interaction network on excellence of life or life satisfaction of these patients. Thus data of 2010 NHIS Sample Adult Cancer' and Quality of Life' were merged for the analysis. We extracted social network properties of the respondent (or the ego') such as personal network size (the number of ego's alters), density (the degree to which alters know each other),nboundedness (the degree to which alters come from different categories of familiarity, such as relatives or neighbor) and centralization (the degree to which network connectedness is dependent upon only a few contacts).

In terms of data collection, this study adopted the egocentric approach, focusing on social relations and interactions that surround cancer patients rather than the whole network analysis and measuring structural pattern of interactions among all adult cancer patients in the dataset and their interaction networks. In the egocentric approach, the focus is to measure patterns of interactions between individuals and how these patterns affect outcomes [8]. In this case, an ego is a particular patient (or a case in the NHIS dataset) while medical doctors, nurses, telephone helpline, friend, family members, people from religious or cancer support communities whom an adult cancer patient associates with are the alters. To develop the alter to alter' ties (or connections between contacts) in each ego network four assumptions were made. For instance a patient who responded YES to the questions Received professional counseling/joined support group after cancer diagnosis" and Participated in research study/clinical trial as part of cancer treatment" have both of these contact types as an alter in the ego network. Information about research studies is usually distributed by health professionals and groups which are working in the same field. Generally, supporting groups and professionals who directly deal with cancer patients, share information about research studies with patients or encourage them to participate in clinical trials or research studies. Thus alter-to-alter connections were developed on the basis of discussion between these two types of supporting contacts in the patient's egocentric network. Other assumptions are listed as below:

- Telephone helplines are commonly a toll free number, which provide basic information to callers. In most cases they refer individuals to contact community or medical centers, which provide more professional and one-on-one counseling services. Thus patients who have both telephone helpline and one-on-one counseling service in their interaction network, have alter to alter connection between these two types of social support variables.

- Patients who answered YES to both questions asking if they Ever had Pap Smear/Pap test" and Đoctor recommended Pap test" are assumed to have alter to alter connection between their social support contacts as Pap test is commonly done by the doctor who recommended doing the test or doctor's assistant nurse.

- Health professional, doctor or nurse who provides information on how cancer may impact emotions and relationship of patients with other people, normally refer the patient to join supporting groups or supply information on how the patient can find supporting groups or professional counseling to cope with the sickness. Thus those patients who answered YES to both questions Health professional, doctor or nurse spoke about how cancer may affect emotions and relationships with other people" and -Receied professional counseling/joined support group to help patient to cope with the illness after

\section{Volume 4 Issue 11, November 2015}




\section{International Journal of Science and Research (IJSR) \\ ISSN (Online): 2319-7064 \\ Index Copernicus Value (2013): 6.14 | Impact Factor (2014): 5.611}

cancer diagnosis" have alter to alter connection between these two types of social support variables.

The relationship between a patient and interaction network members was assumed to be directional. This assumption was made on the basis that patients contact health professionals to seek help or advice. It is very rare for health professionals to contact patients unless another type of relation apart from doctor and patient exist, such as friendship, colleague or family relationship. As we don't have any information about interaction network members to locate if there is a friendship relation between a patient and health professional members thus, it was assumed that connections are initiated by patients and relationship's directions are from patient's side.

\section{Egocentric Network Measures}

Density: The density of a binary network is basically the ratio of all possible ties that are in fact present. It also describes the amount of ties divided by the number of possible ties in a valued network (for instance the proportion of all tie strength that is essentially existent to the number of possible ties). Foran undirected graph with $\mathrm{n}$ nodes, density $\mathrm{D}$ is defined as:

$$
D=\frac{\sum_{i, j=1}^{n} X_{i j}}{n(n-1) / 2}
$$

Where $\mathrm{Xij}$ is the value of the connection from $\mathrm{i}$ to $\mathrm{j}$

Degree Centrality: Freeman [18] defined degree centrality as the count of the degree or number of adjacencies for a point, $\mathrm{P}_{\mathrm{k}}$. Where $\left(\mathrm{P}_{\mathrm{i}}, \mathrm{P}_{\mathrm{k}}\right)=1$ if and only if $\mathrm{P}_{\mathrm{i}}$ and $\mathrm{P}_{\mathrm{k}}$ are connected by a line, 0 otherwise"

$$
\mathrm{C}_{\mathrm{D}}\left(\mathrm{P}_{\mathrm{k}}\right)=\sum_{\mathrm{i}=1}^{\mathrm{n}} \mathrm{a}\left(\mathrm{P}_{\mathrm{i}}, \mathrm{P}_{\mathrm{k}}\right)
$$

Tie Strength: Tie strength of patients is measured as number of times (frequency of contact) that each patient was in contact with people in interaction network and health professionals, who were determined based on NHIS survey and responses in the dataset. The interaction network was defined based on the instrument's variable name and responses. Then unique codes were assigned to each of those interaction network members.

Frequency of contact indicates strength of the tie between actors (each patient) and alters.

Functional Diversity: Diversity index which is a quantitative measure indicates how diverse a dataset is and simultaneously how basic entities are distributed among those diverse types. The functional diversity index was calculated using Shannon's diversity index which was initially proposed by -Claude Shannon" to measure the entropy (uncertainty or information content) in strings of text [44].

$$
H=-\sum_{i=1}^{s} P_{i}\left(\ln , P_{i}\right)
$$

Where if there are $\mathrm{N}$ possible states in which the system can be, $\mathrm{P}_{\mathrm{i}}$ is the probability that the system will be found in state $\mathrm{i}$, the only exception being when the a state is not represented in the case of which the value is 0 [1].

Consequently, if an actor (patient) has ties to professionals from diverse occupations, her/his functional diversity would be considered high. In this study, social support variables were grouped and categorized into family, health professional, community and other for analyzing functional diversity. The health professional category includes nurse, medical doctor or dentist. Equipment or services which help patients to cope with their situation or fulfill their physical needs such as wheelchair or telephone helpline are categorized as $\Theta$ ther"

Efficiency: Efficiency models the effective size of ego's network by its actual size and is measured by dividing effectiveness by the number of alters in the ego's network. It indicates proportion of ego's ties to its neighbourhood which is non-redundant. Furthermore, Efficiency represents the amount of impact ego is getting for each unit participated in using ties. The effective size of the network is described as:

$$
\sum_{j}\left[1-\sum_{q} P_{i q} m_{j q}\right] q \neq i, j
$$

Where $\mathrm{i}$ is the ego, actor $\mathrm{j}$ is a primary contact, and actor $\mathrm{q}$ is also a primary contact who has strong ties with the ego $i$ (represented by $\mathrm{P}_{\mathrm{iq}}$ ) and actor $\mathrm{j}$ (represented bym $\mathrm{j}_{\mathrm{jq}}$ )

Constraint: Ego constraint measures the opportunities held back by the extent to which the ego has invested time and energy in relations with alters that lead back to a single contact [8, p. 55]. In other words, it measures the extent to which the ego's connections are to others who are connected to one another. Constraint on an actor's network is defined as:

$$
\left(\mathrm{P}_{\mathrm{ij}}+\sum_{\mathrm{q}} \mathrm{P}_{\mathrm{iq}} \mathrm{P}_{\mathrm{qj}}\right)^{2}, \mathrm{q} \neq \mathrm{i}, \mathrm{j}
$$

Where $\mathrm{i}$ is the ego, actor $\mathrm{j}$ is a primary contact, and actor $\mathrm{q}$ is also a primary contact who has strong ties with the ego $\mathrm{i}$ (represented by $\mathrm{P}_{\mathrm{iq}}$ ) and actor $\mathrm{j}$ (represented by $\mathrm{P}_{\mathrm{qj}}$ )

\section{Conclusions}

Methodologically, we have demonstrated that it is possible to extract relational data from traditional social surveys and census data to provide further insights into the association between social network properties of the individual and their individual attributes such as Mental QOL. This, we believe, is a significant contribution, which network science academics, industry professionals and organizational network analysts can benefit from. With abundance of census data collected from nations world-wide, this research provides ways forward for eliciting and analysing social network data from social surveys. At the domain level, we believe that this study will generate huge interest and meaningful dialogue in the healthcare community, particular in cancer care, cancer coordination, health services and the quality of life community. The study provides a novel way of studying Mental QOL through social network analysis using a 


\section{International Journal of Science and Research (IJSR) \\ ISSN (Online): 2319-7064 \\ Index Copernicus Value (2013): 6.14 | Impact Factor (2014): 5.611}

significant national survey such as the NHIS and provides springboard for useful policies and interventions to be made.

With the popularity of social media, large numbers of people use online communities to connect to society, nurturing the novel concept of online social capital. We aimed to study the characteristics of people in different social capital. We established a framework of how social media can be used as a barometer for health, and how we can use the underlying statistics to predict future health conditions. We addressed the formulation of online social capital, and establish the link between online social capital defined from social connectivity and users' health. Social media, indeed, can be a barometer of health. And, if used wisely, can play an important part in monitoring well-being.

\section{References}

[1] Ancona, D. G. and Caldwell, D. F. 1992. "Demography and Design: Predictors of New Product Team Performance." Organization Science 3(3): 321-341.

[2] Antonovsky A (1979) Health, stress, and coping. JosseyBass, San Francisco

[3] Berkman LF, Syme SL. 1979. Social networks, hostresistance, and mortality-9-year follow-up study of Alameda County residents. Am. J. Epidemiol. 109(2):186-204

[4] Berkman, L. F., Glass T., Brissette I., Seeman T. E. 2000. From social integration to health:Durkheim in the new millennium, Soc. Sci. Med. 51(6):843-57

[5] Blazer D. 1982. Social support and mortality in an elderly community population. Am. J. Epidemiol.115:684-94

[6] Broadhead, W. E., Kaplan, B. H., James, S. A., Wagner, E. H.,Schoenbach, V. J., Grimson, R., Heyden, S., Tibblin, G., \& Gehlbach, S.H. (1983). The epidemiologic evidence for a relationship between socialsupport and health. American Journal of Epidemiology, 117, 521-537.

[7] Burckhardt, C. S. \& ANDERSON, K. L. 2003. The Quality of Life Scale(QOLS): Reliability, Validity, and Utilization. Health and quality of life.

[8] Burt, R. S. 1992. "Structural holes: The social structure of competition.".

[9] Burt, R. S. 2004. "Structural holes and good ideas." American Journal ofSociology 110(2): 349 - 399.

[10] Burt, R. 2005. "Brokerage and Closure: An Introduction to SocialCapital " Oxford University Press.

[11] Calman, K. C. 1984. Quality of life in cancer patients-an hypothesis.medical ethics, 10, $124-127$.

[12] Cassel J. 1976. Contribution of social-environment to host-resistance- 4thWade Hampton Frost Lecture. Am. J. Epidemiol. 104(2):107-23

[13] Cobb S. 1976. Social support as a moderator of life stress. Psychosom. Med. 38(5):300-14

[14] Cohen S, Syme SL (1985) Social support and health. Academic Press, New York

[15] Cross, R. and J. N. Cummings 2004. "Tie and network correlates of individual performance inknowledge_intensive work." The Academy of Management Journal 47(6): 928_937.

[16]Dakof, G. A. and Taylor, S. E. 1990. Victims' perceptions of social support: what is helpful from whom? Journal of personality and socialpsychology, ISSN 0022-3514, 01/1990, Volume 58, Issue 1, pp. 80 89

[17]Delbrück H (2008). Social support in cancer rehabilitation andpalliation. Rehabilitation and palliation of cancer patients. 51-63.available at: http://www.springerlink.com/content/g6178286w814148 6/

[18]Felce, D. and J. Perry (1995). "Quality of life: Its definition and measurement." Research in Developmental Disabilities 16(1): 51 - 74.

[19] Freeman, L. C. (1978). "Centrality in social networks: Conceptual clarification." Social Networks 1(3): 215239.

[20]Friedkin N. E. 1993 Structural Bases of Interpersonal Influence in Groups: A Longitudinal Case Study, American Sociological Review, ISSN 0003-1224, 12/1993, Volume 58, Issue 6, pp. 861 - 872

[21]Friedman LC, Kalidas M, Elledge R, et al (2005). Optimism, social support and psychosocial functioning among women with breast cancer. Psychooncology, 15 , 595-603.

[22] Gotay C, Wilson ME (1998). Social support and cancer screening in African American, Hispanic, and Native American women.Cancer Practice, 6, 31-7.

[23] Granovetter, M. S. (1973). "The strength of weak ties." American Journal of Sociology 78(6): 1360-1380.

[24] Gruenewald , T. L. , \& Seeman , T. E. ( 2010 ). Social support and physical health: Links and mechanisms . In A. Steptoe (Ed.), Handbook of behavioral medicine (pp. $224-236$ ). New York, NY : Springer Science

[25] Hanneman, R. A. (2001). Introduction to Social Network Methods. Retrieved 8th,August, 2004, from http://faculty.ucr.edu/ hanneman/SOC157/NETTEXT.P DF

[26] Hirsch, B. J. 1980. Natural support systems and coping with major life changes. Am. J. Commun. Psychol. 8(2):159-72

[27] Hirsch, B. J. 1981. Social networks and the coping process. In Social Networks and So-cial Support, ed. B. H. Gottlieb, pp. 149- 69. Beverly Hills: Sage

[28]House J (1981) Work stress, and social support. AddisonWesley, Reading, Mass

[29] House JS, Robbins C, Metzner HL. 1982. The association of social relationships and activities with mortality: prospective evidence from the Tecumseh Community Health Study. Am. J.Epidemiol. 116:123-40

[30] House JS, Landis KR, Umberson D. 1988. Social relationships and health. Science 241:540-45

[31] Kadushin, Charles. 1981. "Notes on Expectations of Reward in NPerson Networks." Pp. 235-54 in Continuities in Structural Inquiry, edited by Peter Blau and Robert Merton. Beverly Hills, Calif.: Sage.

[32] Katapodi M, Facione NC, Miaskowski C, et al (2002). The influence of social support on breast cancer screening in a multicultural community sample. Oncology Nursing Forum (ONF), 29, 845-52.

[33] Kroenke C. H., Kubzansky L. D., Schernhammer E. S., Holmes M. D., and Kawachi I., 2006, Social Networks, Social Support, and SurvivalAfter Breast Cancer Diagnosis." 